\title{
On the Reliability of Eddy Viscosity Based Turbulence Models in Predicting Turbulent Flow past a Circular Cylinder Using URANS Approach
}

\author{
B. N. Rajani ${ }^{1 \dagger}$, A. Kandasamy ${ }^{2}$ and S. Majumdar ${ }^{3}$ \\ 1 Scientist, CTFD Division, National Aerospace Laboratories(CSIR), Bangalore, 560017 India \\ 2 Professor, DMACS, National Institute of Technology Karnataka, Surathkal 575 025, India \\ 3 Professor, Dept. of Mech. Engg., NITTE Meenakshi Institute of Technology, Bangalore 560 640, India \\ $†$ Corresponding Author Email: rajani@ctfd.cmmacs.ernet.in
}

(Received March 8, 2009; accepted March 28, 2009)

\begin{abstract}
Turbulent flow past circular cylinder at moderate to high Reynolds number has been analysed employing an secondorder time accurate pressure-based finite volume method solving two-dimensional Unsteady Reynolds Averaged Navier Stokes (URANS) equations for incompressible flow, coupled to eddy-viscosity based turbulence models. The major focus of the paper is to test the capabilities and limitations of the present turbulence model-based 2D URANS procedure to predict the phenomenon of Drag Crisis, usually manifested in reliable measurement data, as a sharp drop in the mean drag coefficient around a critical Reynolds number. The computation results are compared to corresponding measurement data for instantaneous aerodynamic coefficients and mean surface pressure and skin friction coefficients. Turbulence model-based URANS computations are in general found to be inadequate for correct prediction of the mean drag coefficients, the Strouhal number and also the coefficients of maximum fluctuating lift over the range of flow Reynolds number varying from $10^{4}$ to $10^{7}$.
\end{abstract}

Keywords: Turbulent flow, Circular cylinder, Drag crisis, Unsteady RANS procedure, Eddy viscosity based turbulence models, Implicit finite volume method

\section{NOMENCLATURE}

$\begin{array}{lll}b_{k}^{i} & \text { projection area of the cell face } & S U \text { and } S P \\ C_{p} & \text { non-dimensional pressure coefficient } & \mathbf{T} \\ C_{d} & \text { drag coefficient } & \left\langle U_{i}\right\rangle \\ C_{l} & \text { lift coefficient } & y+ \\ D & \text { cylinder diameter } & \beta \\ f & \text { frequency } & \Delta t \\ J & \text { Jacobian of transformation matrix } \mathbf{T} & \Delta v \\ k & \text { turbulent kinetic energy } & \varepsilon \\ S t & \text { Strouhal number } & \eta_{k}^{i} \\ \langle P\rangle & \text { phase-averaged pressure } & \phi \\ R e & \text { flow Reynolds number based on } D & \mu_{t}\end{array}$

\section{INTRODUCTION}

Flow around a circular cylinder is a challenging kaleidoscopic phenomenon. Cross flow normal to the axis of a circular cylinder and the associated problems of heat and mass transport are encountered in a wide variety of engineering applications. Both measurements and computations for flow past circular cylinder over a 
known in literature as the Von Karman vortex street. The laminar vortex shedding is observed to be continuing up to a value of $R e$ of about 190 , beyond which the two dimensional flow becomes unstable and three dimensional, eventually leading to the simultaneous formation of spanwise and streamwise vortex structures. These three-dimensional disturbances propagate further downstream and the flow in the thin free shear layer bordering the far wake zone undergoes slow transition from laminar to turbulent state. As the Reynolds number is further increased, the transition inception location moves further upstream and alters even the structure and evolution of the vortex shedding in the near wake zone.

At around $R e=2 \times 10^{5}$, the boundary layer on the cylinder surface is observed to becomes unstable and the transition point reaches close to the laminar separation point on the cylinder surface. Eventually the transition takes place in the boundary layer which slowly turns to be turbulent (Achenbach 1968; Roshko 1961). At the critical Reynolds number, when the laminar to turbulent transition takes place in the boundary layer at a location somewhere before the laminar separation point, the turbulent boundary layer is able to sustain the adverse pressure gradient for a longer distance and hence the separation point is shifted further downstream. As a consequence of such downstream shift of the separation point, the base pressure on the rear part of the cylinder is increased and hence the drag is observed to be drastically reduced. The Reynolds number at which this phenomenon of the so-called 'Drag Crisis' occurs is known as the critical Reynolds number, which however depends to a great extent on the other disturbances of the experimental situation. Measurement data reported by various researchers (Zdravkovich 1997; Achenbach 1968; Roshko 1961) have shown that the value of the drag coefficient reduces from approximately 1.2 at the Subcritical Reynolds number range to about 0.3 in the Supercritical flow regime. It is also worthwhile to note from the measurement data that as the Reynolds number is increased further to the transcritical flow regime $\left(R e>3.4 \times 10^{6}\right)$, the drag coefficient once again increases slowly to about 0.7 . As the Reynolds number reaches the range of $R e=10^{7}$, the transition inception point is shifted further upstream to a location very close to the front stagnation point and the flow is fully turbulent almost everywhere around the cylinder and in the wake as well.

The challenging flow past circular cylinders have always been a very interesting and exciting research topic for the experimental fluid dynamicists, leading to a large volume of measurement data on flow past cylinders. On the other hand, in the area of numerical simulation of time-dependent three-dimensional flow around circular cylinder, very few research results (Rajani et al. 2009; Kakuda et al. 2007; Mittal 2001; Mittal and Balachandra 1997; Karniadakis and Triantafyllou 1992; Tamura et al. 1990) have been reported in the recent years. Moreover most of these detailed three-dimensional flow computations are limited only to laminar range at very low Reynolds number of the order of 200-300, where the objective is only to demonstrate the appearance of three- dimensional vortical flow structures and the chaotic state of flow in the cylinder wake even at a Reynolds number as low as 300 or so. As the Reynolds number increases, the phenomena of transition and turbulence need to be appropriately simulated for a realistic simulation of the flow situation. In spite of being the most accurate simulation methodology, Direct Numerical Simulation approach, under the present constraints of computation resources, is prohibitively expensive for higher Reynolds number flows consisting of very wide range of length and time scales.

The literature on the simulation of turbulent flow past circular cylinder at high Reynolds number using URANS methodology coupled to turbulence models or using LES methodology are also quite limited. LES computations are reported using either Smagorinsky (Selvam 1997; Song and Yuan 1990) type sub grid scale models or using no turbulence models as such (Singh and Mittal 2005; Kashimaya et al. 1998; Kakuda and Tosaka 1992; Tamura and Kuwahara 1989) to simulate the turbulent interactions at the sub grid level. Deng et al. (1993) have used the algebraic BaldwinLomax model as well as the $k-\varepsilon$ turbulence models to study the phenomenon of drag crisis, whereas advanced turbulence models like SST and SA have been used by Cox (1997) for similar two-dimensional flow simulations. Most of the computational results available in literature have usually over predicted the mean drag in the subcritical regime compared to the corresponding measurement data.

The objective of the present URANS computation is mainly to assess the limitation and accuracy level of the existing popular and widely used turbulence models for computation of aerodynamic drag and specially in predicting the phenomenon of Drag Crisis for flow past a circular cylinder over a certain range of flow Reynolds numbers. However the present computations are restricted to two-dimensional flow only since measurement data and other computation results reported in literature (Singh and Mittal 2005; Williamson 1996; Braza et al.1990) confirm that the free shear layer instability in the wake which happens to be the prime source of laminar to turbulent transition and eventually leads to drag crisis, is a two-dimensional phenomenon only. Computation-intensive threedimensional flow calculation using turbulence modelbased URANS methodology will be meaningful only if the turbulence models, even for two-dimensional flow computation, can mimic the physics of the transitional flow with reasonable accuracy.

In the present two dimensional numerical simulation, computations have been carried out using different turbulence models for a range of Reynolds number covering the subcritical to the supercritical regime $(R e$ ranging from $10^{4}$ to $10^{7}$ ). All the computations have been carried out using an implicit finite volume type Navier Stokes code RANS3D, developed at the CTFD Division, NAL, Bangalore, India (Majumdar et al. 1992; Majumdar 1998; Majumdar et al. 2003). The computation results have been validated against measurement and other computation data reported in the literature. 


\section{Mathematical Modeling OF Flow PHYSICS}

The analysis of flow behind a circular cylinder over a wide range of flow Reynolds number has been carried out in the present work through numerical solution of the relevant Unsteady Reynolds Averaged Navier Stokes (URANS) equation system, coupled to four different eddy viscosity based turbulence models. The basic equations to be solved are a set of non-linear, strongly coupled partial differential equations representing the conservation of mass, conservation of three mean momentum components along the cartesian directions and the conservation of relevant turbulence scalars for evaluation of the Reynolds stresses.

\subsection{Unsteady Reynolds Averaged Navier Stokes (URANS) Equations}

In the URANS approach, the Reynolds averaging concept, is directly used to replace the instantaneous flow variables by the so-called time-averaged variables or phase-averaged variables for the steady and timedependent mean flow situations respectively. The only assumption in this representation is that the time scale of the mean flow variation has to be quite large compared to the time scale of the turbulent fluctuations. The effect of turbulent fluctuation on the mean flow is therefore represented in the mean momentum equations in the form of a second moment correlation between the unknown fluctuating velocity components. The cartesian coordinate system $\left(y_{1}, y_{2}, y_{3}\right)$ are transformed to the non-orthogonal coordinate system $\left(x_{1}, x_{2}, x_{3}\right)$ using the chain rule of differentiation as follows :

$\left[\frac{\partial}{\partial y_{1}}, \frac{\partial}{\partial y_{2}}, \frac{\partial}{\partial y_{3}}\right]^{t}=\mathbf{T}\left[\frac{\partial}{\partial x_{1}}, \frac{\partial}{\partial x_{2}}, \frac{\partial}{\partial x_{3}}\right]^{t}$

where $\mathbf{T}$ is the transformation matrix and $T_{i j}=\frac{\partial y_{j}}{\partial x_{i}}$.

The URANS equations for turbulent incompressible flow in non-orthogonal curvilinear coordinates with cartesian velocities as dependent variables may be written in a compact form as follows :

Momentum transport for the Cartesian velocity component $\left\langle U_{i}\right\rangle$ :

$$
\begin{gathered}
\frac{\partial\left(\rho\left\langle U_{i}\right\rangle\right)}{\partial t}+\frac{1}{J} \frac{\partial}{\partial x_{j}}\left[\left(\rho\left\langle U_{i}\right\rangle\left\langle U_{k}\right\rangle \eta_{k}^{j}\right)+\langle P\rangle \eta_{i}^{j}\right. \\
-\frac{\mu}{J}\left(\frac{\partial\left\langle U_{i}\right\rangle}{\partial x_{m}} B_{m}^{j}+\frac{\partial\left\langle U_{k}\right\rangle}{\partial x_{m}} \eta_{i}^{m} \eta_{k}^{j}\right) \\
\left.-\rho\left\langle u_{i} u_{k}\right\rangle \eta_{k}^{j}\right]=S_{U_{i 3}}
\end{gathered}
$$

where, $u_{i}$ is the fluctuating velocity component, $S_{U_{i}}$ is any momentum source other than the pressure gradient, $\eta_{k}^{j}$ are the metric coefficients (cofactor of the term $\frac{\partial y_{j}}{\partial x_{k}}$ in the transformation matrix $\mathbf{T}$ ) and $B_{m}^{j}=\eta_{n}^{j} \eta_{n}^{m} \cdot \eta_{k}^{j}$ is expressed in terms of the projection area of the faces as follows :

$\boldsymbol{\eta}_{k}^{j}=b_{k}^{j} \Delta x_{j} /\left(\Delta x_{1} \Delta x_{2} \Delta x_{3}\right)$ where the areas $b_{j}^{i}$ for the control volume around $P$ are shown in Fig. 1b for a two-dimensional situation on $y_{1}-y_{2}$ plane. These momentum equations are further supplemented by the mass conservation or the so-called continuity equation

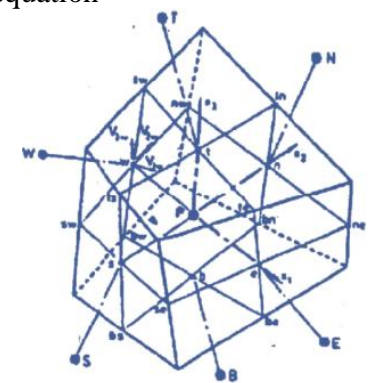

(a) Hexahedral control volume

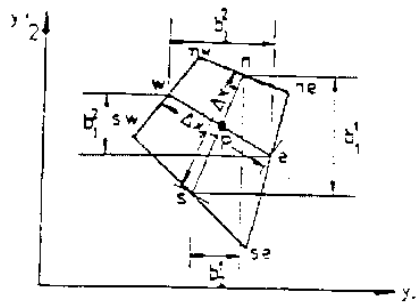

(b) Projection areas for 2D Cell

Fig. 1. Typical control volume and cell face projection area

Mass conservation (Continuity):

$$
\frac{\partial\left(\rho\left\langle U_{k}\right\rangle \eta_{k}^{j}\right)}{\partial x_{j}}=0
$$

However Eq. 1 and Eq. 2 do not form a closed system due to the presence of the unknown turbulent stress term $-\rho\left\langle u_{i} u_{j}\right\rangle$.

\subsection{Turbulence Modeling}

\subsubsection{Eddy Viscosity Hypothesis}

In Eddy Viscosity based turbulence models, the turbulent stress appearing in the Reynolds-Averaged equations is expressed in terms of the mean velocity gradients as following :

$-\rho\left\langle u_{i} u_{k}\right\rangle=\mu_{t}\left(\frac{\partial\left\langle U_{i}\right\rangle}{\partial x_{k}}+\frac{\partial\left\langle U_{k}\right\rangle}{\partial x_{i}}\right)-\frac{1}{3} \rho \delta_{i k}\left\langle u_{m} u_{m}\right\rangle$

where, $\delta_{i k}$ is the Kronecker Delta and $k$ is the summing index over $k=1,2,3$. The eddy viscosity $\mu_{t}$ is assumed to be an isotropic scalar quantity whose value depends on the local state of turbulence. Substituting the turbulent stress term in Eq. 1 and carrying out some algebraic manipulation one may rewrite the mean momentum equation as following:

$$
\begin{gathered}
\frac{\partial\left(\rho\left\langle U_{i}\right\rangle\right)}{\partial t}+\frac{1}{J} \frac{\partial}{\partial x_{j}}\left[\left(\rho\left\langle U_{i}\right\rangle\left\langle U_{k}\right\rangle \eta_{k}^{j}\right)+\langle P\rangle \eta_{i}^{j}\right. \\
\left.-\frac{\left(\mu+\mu_{t}\right)}{J}\left(\frac{\partial\left\langle U_{i}\right\rangle}{\partial x_{m}} B_{m}^{j}+\frac{\partial\left\langle U_{k}\right\rangle}{\partial x_{m}} \eta_{i}^{m} \eta_{k}^{j}\right)\right]=S_{U_{i}}
\end{gathered}
$$

The algebraic or zero equation turbulence models (Baldwin and Lomax 1978; Cebeci and Smith 1974), 
employed very successfully for attached boundary layer type flows, compute the eddy viscosity $\left(\mu_{t}\right)$ at any field point as an algebraic function of the mean velocity gradients and the normal distance from the solid surface.

These models are computationally cheap but sometimes call for complicated interpolations to determine the normal distance from wall for highly skewed grids near the body surface and cannot, in general, simulate separated flows. On the other hand for eddy viscosity based turbulence models, transport equation are solved for one or more turbulence scalars. Four different turbulence models used in the present work are the low Reynolds number version of $k-\varepsilon$ model proposed by Chien (1982), Shear Stress Transport (SST) model proposed by Menter (1994), one equation model of Spalart-Allamaras (SA) (Spalart and Allamaras 1992) and $k-\varepsilon-\overline{v^{2}}-f$ model, popularly known as V2F model, proposed by Durbin (1995).

\subsection{Finite Volume Formulation}

The present flow solution algorithm (RANS3D) is an iterative two-step predictor-corrector procedure similar to the SIMPLE algorithm of Patankar (Patankar 1980). In the predictor step, the momentum equations are solved to advance the velocity field partially in time for a guessed pressure field. The continuity is then ensured by solving a Poisson equation for pressure correction, followed by relevant correction of the pressure and velocity field in the corrector step. Integration of the momentum transport equations (Eq. 4) over each control volume (Fig 1a) transforms the relevant pde's in the form of discrete algebraic equations representing a balance between the convective and diffusive fluxes through the cell faces and the other remaining terms as volume sources.

Second order accurate central difference scheme is used for spatial discretisation of the convective and diffusive fluxes at the cell faces. The temporal derivatives are also discretised using the second order accurate threelevel fully implicit scheme. The numerical stability is ensured by means of a deferred correction procedure (Khosla and Rubin 1974) where a suitable weighting function is used to blend the flux from the desired scheme with upwind fluxes which allow some small numerical diffusion for stability of the solution. Using the relevant geometric factors with second order spatial and temporal discretisation schemes for cell face fluxes and linearisation of the source terms, the flux balance equation is expressed in an implicit manner in the following quasi-linear form:

$$
\begin{gathered}
\left(1.5 \phi_{P}^{n+1}+0.5 \phi_{P}^{n-1}-2 \phi_{P}^{n}\right) \Delta V / \Delta t+A_{P} \phi_{P}^{n+1} \\
=\sum_{n b} A_{n b} \phi_{n b}^{n+1}+S U
\end{gathered}
$$

where $A_{P}=\sum A_{n b}-S P$; the coefficient $A_{n b}$ represents the combined effect of convection and diffusion from the neighbouring nodes at the cell faces; $S U$ and $S P$ are the components of the linearised source term, $\Delta v$ is the cell volume and $\Delta t$ is the time step size. The superscripts of $\phi$ represent the respective time step. The detailed derivation of Eq. 5 in terms of the flow variables and the cell face projection areas is given elsewhere (Majumdar et al. 1992; Majumdar et al. 2003). The transformation of continuity equation to an equation of pressure correction, described in the following subsection. A segregated iterative method, coupled to the strongly implicit procedure of Stone (Stone 1968), is used to solve the system of linear equations (Eq. 5) corresponding to each transport equation in a sequential manner. At each time step, the normalized residue for all the equations solved are brought down to a convergence criterion of $10^{-5}$.

\subsection{Computation of Pressure Field}

Algorithms based on pressure-velocity solution strategy transform the continuity equation into a field equation for pressure correction using momentum equations as link between pressure and velocity corrections. For three dimensional flow analysis using a collocated variable arrangement, the problem of the so-called checkerboard spitting is avoided by employing the principle of Momentum Interpolation (Majumdar 1988; Majumdar et al. 1992). This gives the cell-face velocity components, $U_{i w}$ (as an illustration, at the cell west face ' $w$ ') and hence the link between pressure and velocity correction $U_{i w}{ }^{\prime}$ as :

$$
\begin{aligned}
& U_{i v}=\alpha_{v} \frac{\overline{H_{p}^{i}+D_{2}^{i}\left(p_{s}-p_{n}\right)+D_{3}^{i}\left(p_{b}-p_{t}\right)}+}{\overline{\left.D_{1}^{i}\left(p_{w}-p_{p}\right)\right]+\left(1-\alpha_{v}\right) U_{i w}^{0}}} \\
& U^{\prime}{ }_{i w}=\alpha_{v} \overline{D_{1}^{i}}\left(p_{W}^{\prime}-p_{P}^{\prime}\right)
\end{aligned}
$$

where $H_{P}^{i}=\left(\sum A_{n b}^{i} U_{i n b}+S U\right) / A_{P}^{i}$ and $D_{k}^{i}=b_{k}^{i} / A_{P}^{i} A_{n b}^{i}$ represents the coefficients for neighbouring nodes, the superscript ' 0 ' is the value at the previous iteration, $A_{P}^{i}$ is the value of $A_{P}$ for $U_{i}$ at the node $P$ (Fig. 1a), $b_{k}^{i}$ is the relevant projection area of the cell face (Fig. 1b), $\alpha_{v}$ is an under-relaxation parameter and the expressions with overbar represent the linear average of the same quantities evaluated at the cell-centers $P$ and $W$ adjacent to the face ' $w$ '. Now the conservation of mass in a control volume may be expressed as:

$C_{e}-C_{w}+C_{n}-C_{s}+C_{t}-C_{b}=0$

where the face mass flux $C_{w}$, for example, may be written as follows using ' $i$ ' as the summation index for three directions.

$C_{w}=\rho_{w} b_{i w}^{1}\left(U_{i w}+U_{i w}^{\prime}\right)$

Substituting $U_{i w}$ and $U_{i w}^{\prime}$ for all ' $i$ ' from equations similar to Eqs. 6 and 7 respectively in mass flux expression similar to Eq. 9 for each of the six faces in Eq. 8, one obtains the equation of pressure correction in a form very similar to Eq. 5. Once the field of pressure correction is solved, corrections are applied to the cellcentre pressure and velocities, to all the cell-face velocities and finally to the convective mass-fluxes to satisfy the cell wise continuity.

\section{RESULTS AND DISCUSSION}

\subsection{Computational Details}

The computational domain consists of the annular region between two circles of diameter $D$ and $40 D$ where $D$ is the diameter of the cylinder and the number 
of nodes used is 144 along the radial and 121 along the circumferential direction divided into two blocks overlapped with one control volume on either side of the block interface. In order to handle a twodimensional problem by the three-dimensional flow solver RANS3D, only two parallel planes are considered along the axial or spanwise direction $(z)$ with the same $2 D$ curvilinear grid stacked on each of the two $z$ planes. At the spanwise end planes Symmetry conditions are applied to ensure two dimensionality of the flow. The two block radial polar grid (Fig. 2a) is used on the two-dimensional plane of interest, between the cylinder of unit diameter $(D)$ and the far field of radius twenty units. The grid lines are stretched radially near the wall to resolve the sharp local gradients of the flow variables. Figure $2 \mathrm{~b}$ shows the boundary conditions used for the turbulent flow computation.

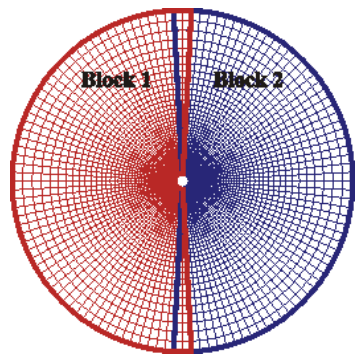

(a) 2-block grid arrangement

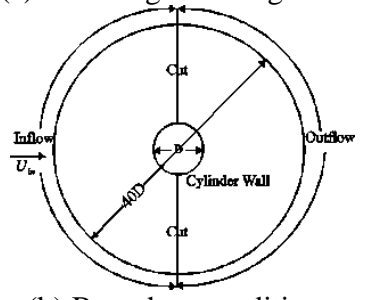

(b) Boundary conditions

Fig. 2. Multiblock polar grid $(124 \times 144)$ and boundary conditions used for computation of flow past circular cylinder

Block 1 consists of an inflow boundary, a wall boundary for the half cylinder and two cut boundaries separating this block from the neighbouring Block 2 as shown in the figure. Similarly, Block 2 consists of an outflow, a wall boundary for the rest half of the cylinder and the two cuts. An impulsive start of the cylinder is simulated by specifying uniform inflow velocity $(U=$ $U_{\infty}$ and $\left.V=0\right)$ at all nodes with no slip conditions $(U=0$ and $V=0$ ), imposed on the cylinder wall as initial conditions $(t=0)$ and maintained thereafter at all time instants $(t>0)$. For the turbulence scalars, appropriate near wall boundary conditions have been used depending on the turbulence model chosen. The level of free stream turbulence energy $(k)$ is maintained at $1 \%$ of the mean kinetic energy of the freestream whereas the value of the turbulence energy dissipation $(\varepsilon)$ and the specific dissipation $(\omega)$ at the inflow boundary nodes is prescribed assuming the local eddy viscosity $\left(\mu_{t}\right)$ to be approximately equal to the laminar viscosity $(\mu)$. In the V2F model, at the inflow boundary nodes the velocity scale $\overline{v^{2}}$ is assumed to be two-thirds of the inlet turbulence kinetic energy $(k)$ and the redistribution function $f$ is set zero. For the SA model the inlet value of $\tilde{v}$ is assumed to be equal to the laminar kinematic viscosity. Effect of the spatial and temporal discretisation schemes and the effect of far field location have earlier been studied in detail for laminar flows. In the present study extensive parametric analysis have been carried out for turbulent flows at $R e$ $=10^{5}$ and $R e=3.6 \times 10^{6}$ in order to study the effect of grid-size, time step size and local eddy viscosity level at the inflow boundary. Based on the previous and present studies, central difference scheme for spatial discretisation coupled to second order time discretisation with $\Delta t=0.05$ have been used for all the turbulent flow computation at different Reynolds number. For very high values of $R e$, the number of grid lines and the near wall grid spacing are so adjusted that the value of $y^{+}$at the first near wall point is maintained to be less than unity for all the turbulence models used.

\subsection{Temporal Evolution of Lift and Drag Coefficients}

The typical temporal evolution of the lift $\left(C_{l}\right)$ and drag $\left(C_{d}\right)$ coefficients for the cylinder computed for Reynolds number of $10^{5}$, using different turbulence models are shown in Fig. 3. The variation of the aerodynamic coefficients are plotted against nondimensional time $\left(\bar{t}=\frac{U_{\infty} t}{D}\right)$. Irrespective of the turbulence model used, the figures clearly demonstrate the phenomenon of periodic fluctuation of the lift and drag coefficient due to turbulent vortex shedding. In each computation however, large number of time steps are required for the initial transients to die out and finally when a statistically stationary state is reached, the number of iterations required per time step is reduced. The temporal variation of the lift $\left(C_{l}\right)$ coefficient shows a periodic fluctuation about the zero mean as expected physically for a symmetric mean flow and the corresponding Fourier spectra (not shown in any figure) indicates a dominant frequency $(f)$, expressed in non-dimensional form as the Strouhal number $\left(S t=\frac{f D}{U_{\infty}}\right)$. In case of $\operatorname{drag}\left(C_{d}\right)$ the Strouhal number for $C_{d}$ is observed to be almost double the corresponding value for the $C_{l}$. Such difference in the frequency of the periodic mean motion between the longitudinal and the transverse direction has also been observed and explained in other measurement and computation data reported in unclassified literature (Mittal 2001; Song and Yuan 1990; Drescher 1956). For the same flow Reynolds number of $10^{5}$, the mean value of the drag coefficient and the RMS of the lift coefficient, obtained by different turbulence models, indicated in the same Fig. 3, are observed to be different. The Chien's low Re $k-\varepsilon$ and the SST model appear to be predicting nearly the same value of maximum $C_{l}$ amplitude as 1.0 at the statistically stationary state whereas the SA model consistently under predicts and the V2F model over predicts the same quantity by about 15 to $20 \%$. Comparison to measurement data on mean drag coefficient $\left(C_{d} \approx 1.2\right)$ reported in literature (Achenbach 1968) demonstrates that the predictions by $\mathrm{V} 2 \mathrm{~F}$ model agree reasonably well; Chien and SST models mildly under predict the $C_{d}$ value whereas SA model grossly under predicts this 
value. At $R e=10^{5}$, the experimentally observed laminar flow separation is consistently over predicted by all the four models. However, the predicted location of separation is at around $90^{\circ}$ whereas the corresponding measured value reported by Achenbach (1968) is around $78^{\circ}$. Further the post separation behaviour of the turbulent flow, specially the combined effect of the turbulent free shear layer bordering the wake and the large vortex structure of the near wake itself in the vicinity of the rear surface of the cylinder, is observed to be quite different for the different turbulence models used. Under prediction of mean drag coefficient for flow past circular cylinder, observed in the present computation with some of the turbulence models, has also been reported by other researchers (Cox 1997; Selvam 1997).

Figure 4 shows the temporal evolution of $C_{l}$ and $C_{d}$ at four different Reynolds number using the Chien's low $R e$ version of $k-\varepsilon$ model. The sharp drop in the level of mean drag coefficient is clearly seen in the figure when $R e$ increased from $10^{5}$ to $8.5 \times 10^{5}$, whereas when the $R e$ is further increased to $3.6 \times 10^{6}$ and $10^{7}$ the level of mean drag further decreases but at a much slower rate. The increase of the drag observed in measurement data (Zdravkovich 1997; Achenbach 1968; Schlichting 1968) when the Reynolds number in increased to $10^{7}$ is not captured by the present computation with any of the four turbulence models, even using reasonably high spatial and temporal resolution (Section 3.3). The maximum value of $C_{l}$ is drastically reduced from about 1.0 at $R e=10^{5}$ to approximately 0.3 at $R e=8.5 \times 10^{5}$, but the level of maximum $C_{l}$ remains almost unchanged as the $R e$ is increased further up to $10^{7}$.

\subsection{Surface Pressure and Skin Friction on Cylinder Surface}

The time averaged flow quantities at any field node are computed from arithmetic averaging of the relevant flow variable over at least 50 vortex shedding cycles after the statistically stationary state is reached. Both the surface pressure and the wall shear stress on the cylinder surface are appropriately non-dimensionalised for pressure as $C_{p}=\frac{\left(p-p_{\infty}\right)}{1 / 2 \rho U_{\infty}^{2}}$ and for skin friction as $\beta=\frac{\tau_{\text {wall }}}{1 / 2 \rho U_{\infty}^{2}} \sqrt{\operatorname{Re}}$

Figure 5 shows the circumferential variation of $C_{p}$ and $\beta$ at four different Reynolds number $\left(R e=10^{5}, 8.5\right.$ $\times 10^{5}, 3.6 \times 10^{6}$ and $\left.10^{7}\right)$ using two different grid sizes one with 121 nodes $\left(\Delta \theta=3^{0}\right)$ along the circumferential direction and 144 points in the radial direction $(\Delta r=1$ $\times 10^{-4} \mathrm{D}$ for $\left.\operatorname{Re}=10^{5}\right)$ and the other with 241 nodes $\left(\Delta \theta=1.5^{0}\right)$ along the circumferential direction and 305 points in the radial direction $\left(\Delta r=1 \times 10^{-4} D\right.$ for $R e=$ $10^{5}$ ). However insignificant effect of grid refinement on the circumferential variation of $C_{p}$ and $\beta$, observed in Fig. 5, indicates the results with $121 \times 144$ nodes to be more or less grid-independent. Similarly for time step size also, the results obtained using $\Delta t=0.05$ and 0.005 shown in Figs. 5a and 5c justify the independence of the solution on the time step size for $\Delta t=0.05$.
The effect of the four different turbulence models on circumferential variation of $C_{p}$ and $\beta$ at four different Reynolds number are shown in Fig. 6. Irrespective of the turbulence model used, the computed pressure distribution and the skin friction coefficient agree reasonably well with the measurement data (Jones et al. 1969; Achenbach 1968) for the front segment of the cylinder covering almost one third of the periphery (about $60^{\circ}$ on either side of the front stagnation point) where the flow mostly consists of the development of a laminar boundary layer under a favourable pressure gradient. But beyond the subcritical regime of $R e=10^{5}$, discrepancies are observed between the computed and measured values of the suction peak attained by this accelerating flow. The corresponding peak of the skin friction coefficient are largely over predicted at almost all the flow Reynolds number beyond $10^{5}$. The other major disagreement lies here in the prediction of the separation point location which, compared to the corresponding measurement data, is invariably predicted further downstream for all the flow situations studied. The prominent double peak in skin friction $\beta$ observed in the measurement data at $R e=8.5 \times 10^{5}$, possibly due to the transition taking place well before the separation, has not at all been captured by any of the turbulence models used. However the measurement data for flow at $R e=3.6 \times 10^{6}$ do not exhibit such double peaks of $\beta$ and it is not clear whether flow at this $R e$ is fully turbulent and the transition point falls very close to the stagnation point. However the large over prediction of the single peak magnitude of $\beta$ at the same Reynolds number is not in conformity with the argument of fully turbulent flow everywhere in the boundary layer on the cylinder surface. At $R e=10^{7}$ when the boundary layer is physically expected to be fully turbulent for most part of the cylinder, the base pressure at the rear surface predicted by all the models is found to be close to the measurement data but the magnitude of the suction peak is over predicted with a downstream displacement of the peak location as well. This disagreement which also explains the discrepancy in the value of the aerodynamic coefficients may be attributed to the inadequacy of the eddy viscosity based turbulence models for flow in the presence of laminar to turbulent transition and also to the strong adverse pressure gradient. Perhaps LES or DNS simulations might be more reasonable approaches for resolving such flows with large vertical structures and often with more than one characteristic frequencies.

\subsection{Phenomenon of Drag Crisis}

In order to understand whether the Unsteady 2D RANS computation employing eddy viscosity based turbulence models, is capable of predicting the interesting phenomenon of Drag Crisis for circular cylinders, observed in measurement data for $C_{d}$ against $R e, 2 \mathrm{D}$ computations have been carried out for a wide range of flow Reynolds number spanning from the laminar regime to as high as $10^{7}$. Figure 7 shows the available measurement data (Zdravkovich 1997; Achenbach 1968; Schlichting 1968) on the mean drag coefficient collected from different sources and also the present computation results for laminar flow as well as for turbulent flow situations using variety of turbulence models. 


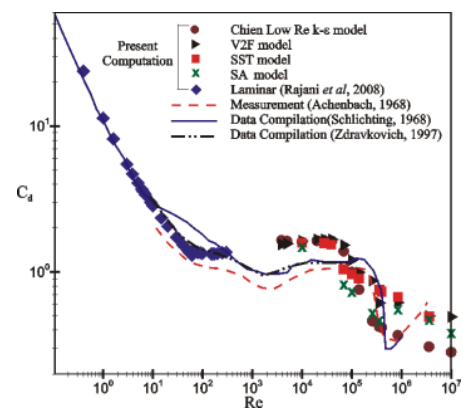

Fig. 7. Effect of turbulence models on the variation of mean drag coefficient with $R e$

(Grid: $121 \times 144, \Delta t=0.05)$

Excellent agreement with the compilation data of Zdravkovich (1997) in the laminar regime up to about $R e=200$ confirms the adequacy of the space and time resolution and also the accuracy of the discretisation schemes used in the flow solution algorithm of RANS3D. Beyond $R e=200$, the important effects of the three dimensional spanwise instabilities on the flow have already been identified by many researchers including the present author (Rajani et al. 2009; Mittal 2001; Mittal and Balachandra 1997; Williamson 1996; Zhang et al. 1995; Williamson and Roshko 1990; Roshko 1955; Roshko 1954). As stated earlier, this complex flow always consists of laminar, turbulent and transitional patches and the discrepancies obviously are expected to be more while using either purely laminar flow equations or fully turbulent flow-based turbulence models for situations with large laminar or transitional patches. Accordingly the zone of $R e$ between 400 and 3000 has been simply omitted for the time being and the unsteady RANS computation has been carried out between $R e=3900$ and $10^{7}$. Up to $R e=6.9 \times 10^{4}$, the turbulence models appear to have insignificant effect on the predicted mean drag coefficient. However beyond $R e=6.9 \times 10^{4}$, a sharp fall in the drag coefficient is observed - but at different rates with different turbulence models. The computation with SA model shows a very sharp fall at $R e=1 \times 10^{5}$ resulting in a strong under prediction of $C_{d}$ compared to the measurement data. The Chien $k-\varepsilon$ model more or less follows the data from other source up to $R e=8.5 \times 10^{5}$, with a sudden drop in $C_{d}$ occurring at $R e=1.4 \times 10^{5}$ which is slightly earlier than that observed in the measurement data. The SST and V2F models also follow a similar trend of the drag variation where the phenomenon of drag crisis occurs at a relatively high value of flow Reynolds number $\left(R e=3.6 \times 10^{5}\right)$ and hence the mean drag coefficient is over predicted for $R e$ $>10^{5}$. However as the $R e$ increases further, results from all the four models more or less follow the same trend of drop in $C_{d}$ till $R e$ reaches about $10^{6}$. Therefore qualitatively a sharp drop in $C_{d}$ in the critical regime is manifested by all the turbulence models, but a wide scatter is observed in the computation results obtained by different models. Further, the falling trend of $C_{d}$ is continued in the prediction even when the Reynolds number is increased to higher value of the order of $10^{7}$. None of the 2D URANS computation is able to capture the rise in $C_{d}$ observed in the measurement data beyond a Reynolds number of $10^{6}$. Figure 8 shows the variation of the mean drag coefficient with flow Reynolds

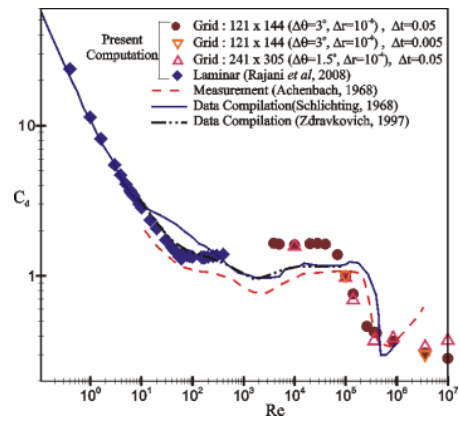

Fig. 8. Effect of grid and time step size on the variation of mean drag coefficient with $\operatorname{Re}$ (Turbulence Model :

Chien low $\operatorname{Re} k-\varepsilon$ )

number using a single turbulence model coupled to different grid and time step sizes.

The overlapping of the data points clearly shows the solution obtained using $121 \times 144$ grid and $\Delta t=0.05$ may be accepted as the grid independent solution and further refinement does not bring any significant effect on the solution. The discrepancies between the measurement data and computation results may mostly be attributed to the inadequacy of the eddy viscosity based turbulence models to capture the transition specially beyond the critical regime when the transition takes place somewhere in the cylinder surface boundary layers and that too at a point upstream of the separation point.

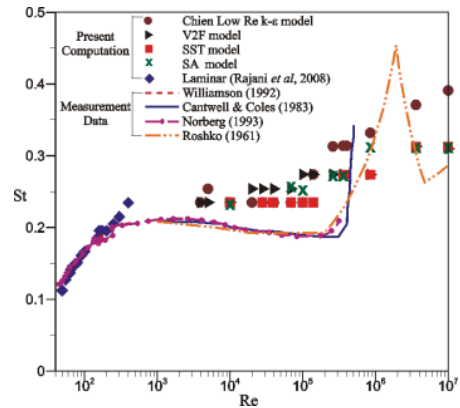

Fig. 9. Effect of turbulence models on the variation of Strouhal number $(S t)$ with $R e$ (Grid:121 $\times 144, \Delta t=0.05)$

Figure 9 compares the computed variation of the Strouhal number $(S t)$ with the flow Reynolds number against measurement data available from different sources (Williamson 1992; Cantwell and Coles 1983; Roshko 1961). Similar to the mean drag variation, the prediction matches reasonably well to measurement data in the pure laminar regime $(R e<250)$. However for turbulent flow regime, the predicted Strouhal number is observed to be almost constant approximately at a level of 0.25 till $R e=10^{5}$ followed by a sharp rise as the value of $R e$ increases towards $10^{7}$. The measurement data for Strouhal number shows a similar trend of variation in a qualitative sense but the approximately constant level of Strouhal number for $10^{3}<R e<3 \times 10^{5}$ is at around 0.2 whereas the predicted level is found to be at the order of 0.25 . Further the peak value of $S t=0.45$ at $R e=2 \times 10^{6}$, followed by a sharp drop, as observed in measurement data of Roshko (Roshko 
1961), could not also be captured by the present computation where instead of the drastic oscillatory behaviour, a slowly increasing trend is clearly observed till $R e=10^{7}$, for all the turbulence models used. The major discrepancies indicate gross inadequacy of the two dimensional flow computation using eddy viscosity based turbulence models for simulation of the present flow situation where the transition taking place in the free-shear layer bordering the near wake zone plays a very important role and eventually the transition is observed to occur along the spanwise direction within the separation bubble itself.

\section{CONCLUding REMARKS}

Two dimensional turbulent flow past a circular cylinder has been computed for flow Reynolds number varying from $10^{4}$ to $10^{7}$ using the Unsteady RANS code RANS3D coupled to four eddy viscosity-based turbulence models. In the subcritical range up to $R e=$ $10^{5}$, all the four turbulence models predict reasonable flow patterns with turbulence vortex shedding at definite frequency (Strouhal number) for a given Reynolds number. The circumferential variation of the time averaged pressure and skin friction around the cylinder for different values of $R e$ show that compared to measurement data, the flow separation location on the cylinder surface is always predicted further downstream, irrespective of whether the separation is laminar or turbulent. As a consequence of that the width of the wake and hence the base pressure at the cylinder rear surface which decides the value of $C_{d}$ is changed. The skin friction coefficient is, in general, largely over predicted for $R e$ beyond the critical regime. This may be attributed to the gross inadequacy of all the turbulence models to capture the effect of laminar to turbulent transition in the boundary layer on the cylinder surface. A sharp fall of the mean drag coefficient $C_{d}$ is captured by all the turbulence models, as observed in measurement; but only the low Reynolds number version of the Chien $k-\varepsilon$ turbulence model is able to predict the phenomenon of drag crisis at a $R e$ closer to the corresponding measurement data. A wide scatter is observed in the predicted variation of the mean drag coefficient with Reynolds number. It is important to note that unlike the measurement data, the falling trend of $C_{d}$ with $R \mathrm{e}$ is not reversed in the prediction for the post or transcritical regime when $R e$ exceeds $5 \times 10^{5}$. The phenomenon of Drag Crisis is captured by the eddy viscosity based turbulence models in a qualitative sense only. More accurate prediction demands realistic simulation of the transition process in the free-shear layer in the subcritical regime and also the complex transition in the boundary layer interacting with the flow separation in the critical and post-critical regime. Work is in progress on Large Eddy Simulation of the same flow, which is likely to capture the physical process of transition through direct numerical simulation of the large eddies and produce more accurate results on this complex unsteady three dimensional flow situation.

\section{ACKNOWLEDGEMENTS}

The authors wish to thank Director NAL Bangalore for his kind permission to publish this paper.

\section{REFERENCES}

Achenbach, E. (1968). Distribution of local pressure and skin friction around a circular cylinder in cross-flow up to $\mathrm{Re}=5 \times 10^{6}$. Journal of Fluid Mechanics 34, 635-639.

Baldwin, B.S. and H. Lomax (1978). Thin layer approximation and algebraic model for separated turbulent flows. AIAA Paper 78, 257.

Braza, M., P. Chassaing, and P.H. Minh (1990). Prediction of large-scale transition features in the wake of a circular cylinder. Physics of Fluid A 2, 1461-1471.

Cantwell, B. and D. Coles (1983). An experimental study of entrainment and transport in the turbulent near wake of a circular cylinder. Journal of Fluid Mechanics 136, 321-374.

Cebeci, T. and A.M.O. Smith (1974). Analysis of turbulent boundary layers. Academic Press, NY.

Chien, K.Y. (1982). Predictions of channel and boundary layer flows with a low Reynolds no. turbulence model. AIAA Journal 20, 321-339.

Cox, J.S. (1997). Computation of sound generated by viscous flow over a circular cylinder. NASA TM 110339.

Deng, G.B., J. Piquet, P. Queutey, and M. Visonneau (1993). Vortex-shedding flow prediction with eddy-viscosity models. Engineering Turbulence Modelling and Experiments, Elsevier Science, Amsterdam 2, 143-152.

Drescher, H. (1956). Messung der auf querangeströmte zylinder ausgeübten zeitlich veränderten drücke. Zeitschrift für Fluggwissenschaften 4, 17-21.

Durbin, P.A. (1995). Separated flows computation with the $k-\varepsilon-\overline{v^{2}}-f$ model. AIAA Journal 33, 659644.

Jones, G.W., J.J. Cincotta, and R.W. Walker (1969). Aerodynamic forces on a stationary and oscillating circular cylinder at high Reynolds numbers. NASA TR R-300.

Kakuda, K., S. Miura, and N. Tosaka (2007). Finite element simulation of 3D flow around circular cylinder. International Journal of Computational Fluid Dynamics 20 (3-4), 193- 209.

Kakuda, K. and N. Tosaka (1992). Finite element approach for high Reynolds number flows. Theoretical and Applied Mechanics 41, 223- 232.

Karniadakis, G.E. and G.E. Triantafyllou (1992). Three-dimensional dynamics and transition to turbulence in the wake of bluff objects. Journal of Fluid Mechanics 238, 1-30. 
Kashimaya, K., T. Tamai, W. Inomata, and S. Yamaguchi (1998). A parallel finite element method for incompressible Navier-Stokes flows based on unstructured grid. $4^{\text {th }}$ Japan-US Symposium F.E.M in Large-Scale Computational Fluid Dynamics, 21-24.

Khosla, P.K. and S.G. Rubin (1974). A diagonally dominant second-order accurate implicit scheme. Computers and Fluids 2, 207-209.

Lienhard, J.H. (1966). Synopsis of lift drag and vortex frequency for rigid circular cylinders. College of Engineering Research Division Bulletin 300, Technical Extension Service, Washington State University.

Majumdar, S., B.N. Rajani, D.S. Kulkarni, and S. Mohan (2003). RANS computation of low speed turbulent flow in complex configuration. Proceedings Symposium on State of the Art and Future Trends of CFD at NAL, NAL SP03 01, 3148.

Majumdar, S., W. Rodi, and J. Zhu (1992). Three dimensional finite volume method for incompressible flows with complex boundaries. Journal of Fluid Engineering, ASME , 496-503.

Majumdar, S. (1988). Role of underrelaxation in momentum interpolation for calculation of flow with non-staggered grids. Numerical Heat Transfer 13, 125-132.

Majumdar, S. (1998). Pressure based Navier Stokes solver for three-dimensional flow in hydrodynamics and low speed aerodynamics application. Proeddings $3^{\text {rd }}$ Asian CFD Conference, Bangalore, 137-146.

Menter, F.R. (1994). Two-equation eddy viscosity turbulence models for engineering application. AIAA Journal 32, 269-289.

Mittal, R. and S. Balachandra (1997). On the inclusion of three-dimensional effects in simulation of twodimensional bluff-body wake flows. ASME Fluids Engineering Division Summer Meeting , 1-6.

Mittal, S. (2001). Computation of three dimensional flow past circular cylinder of low aspect ratio. Physics of Fluids 13, 177-191.

Patankar, S.V. (1980). Numerical heat transfer and fluid flow. Hemisphere Pub. Co.

Rajani, B.N., A. Kandasamy, and S. Majumdar (2009). Numerical simulation of laminar flow past a circular cylinder. Applied Mathematical Modelling $33,1228-1247$.

Roshko, A. (1954). On the development turbulence wakes from vortex streets. NACA Report 1191.
Roshko, A. (1961). Experiments on the flow past a circular cylinder at very high Reynolds number. Journal of Fluid Mechanics 10, 345-356.

Roshko, A. (1955). On the wake and drag of bluff bodies. Aeronautical Science 22, 124.

Schlichting, H. (1968). Boundary Layer Theory. McGraw Hill series in Mechanical Engineering.

Selvam, R.P. (1997). Finite element modelling of flow around a circular cylinder using LES. Journal of Wind Engineering and Industrial Aerodynamics 67 \& 68, 129-139.

Singh, S.P. and S. Mittal (2005). Flow past a cylinder : shear layer instability and drag crisis. International Journal for Numerical Methods in Fluids 47, 75-98.

Song, C.C. and M. Yuan (1990). Simulation of vortecshedding flow about a circular cylinder at high Reynolds number. Journal of Fluids Engineering, ASME 112, 155-163.

Spalart, P.R. and S.R. Allamaras (1992). A oneequation turbulence model for aerodynamic flow. AIAA paper 92-0439.

Stone, H.L. (1968). Iterative solution of implicit approximations of multidimensional partial differential equations. SIAM Journal of Numerical Analysis 5, 530-530.

Tamura, T. and K. Kuwahara (1989). Direct finite difference computation of turbulent flow around circular cylinder. Proc. International Symposium on Computational Fluid Dynamics , 701-706.

Tamura, T., I. Ohta, and K. Kuwahara (1990). On the reliability of two-dimensional simulation for unsteady flow around a cylinder-type structure. Journal of Wind Engineering and Industrial Aerodynamics 35, 275-298.

Williamson, C.H.K. (1992). The natural and forced formation of spot-like dislocations in the transition of a wake. Journal of Fluid Mechanics 243, 393.

Williamson, C.H.K. (1996). Vortex dynamics in the cylinder wakes. Annual Review of Fluid Mechanics 28, 477-539.

Williamson, C.H.K. and A. Roshko (1990). Measurement of base pressure in the wake of a cylinder at low Reynolds numbers. Z. Flugwiss, Weltraumforsch 14, 38-46.

Zdravkovich, M. (1997). Flow around circular cylinders: V.1. Oxford Science Publication.

Zhang, H.Q., U. Fey, B.R. Noack, M. König, and H. Eckelmann (1995). On the transition of the cylinder wake. Physics of Fluids 7, 779-794. 
B.N. Rajani et al. / JAFM, Vol. 5, No. 1, pp. 67-79, 2012.
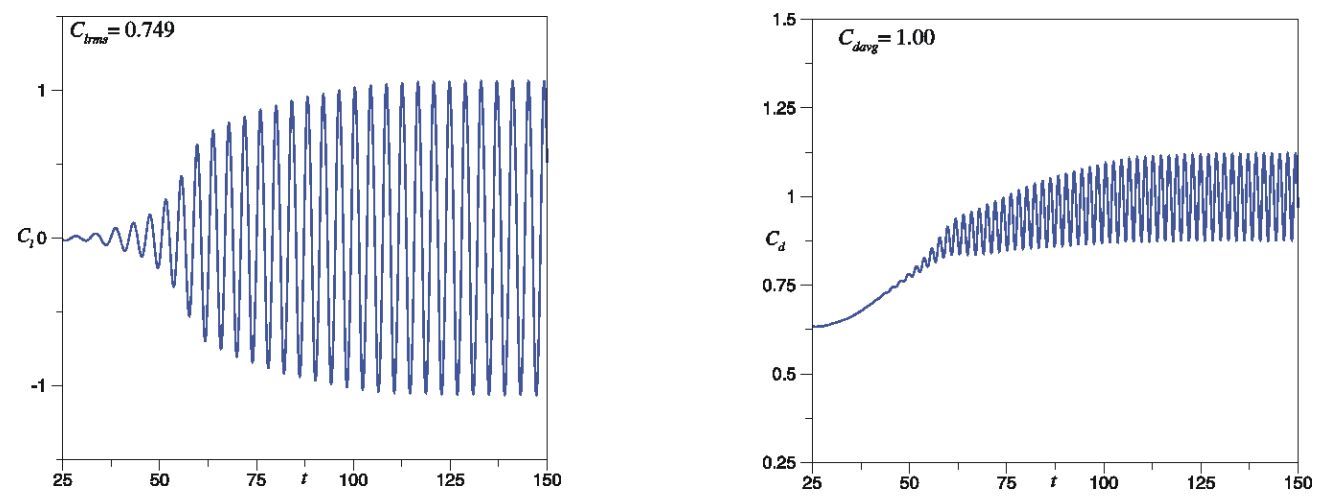

(a) Chien Low Re $k-\varepsilon$ Model
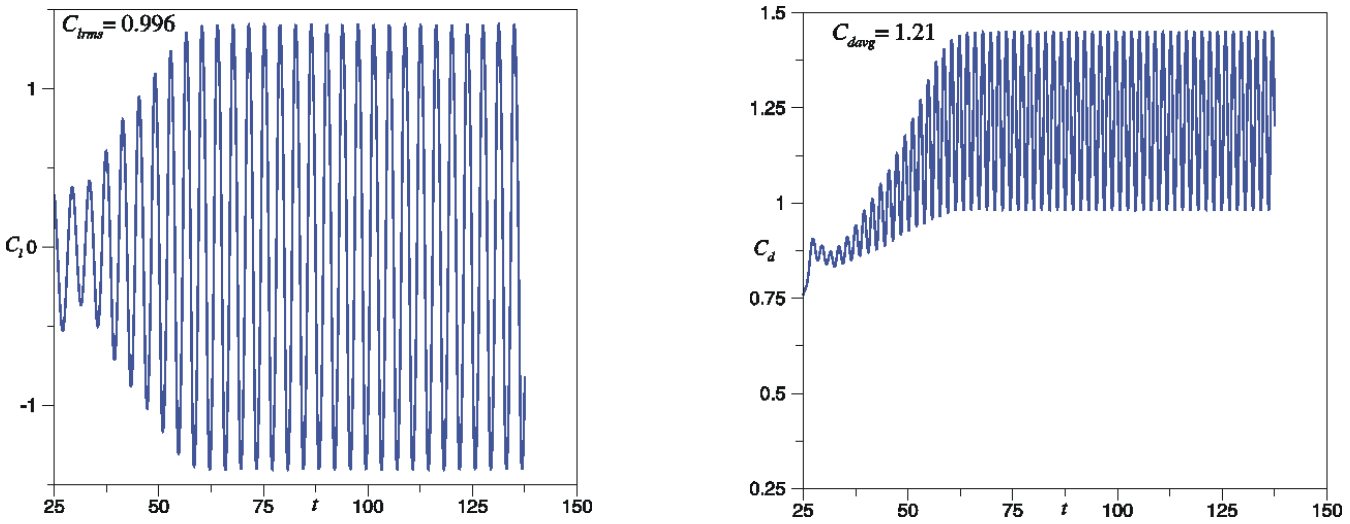

(b) V2F Model
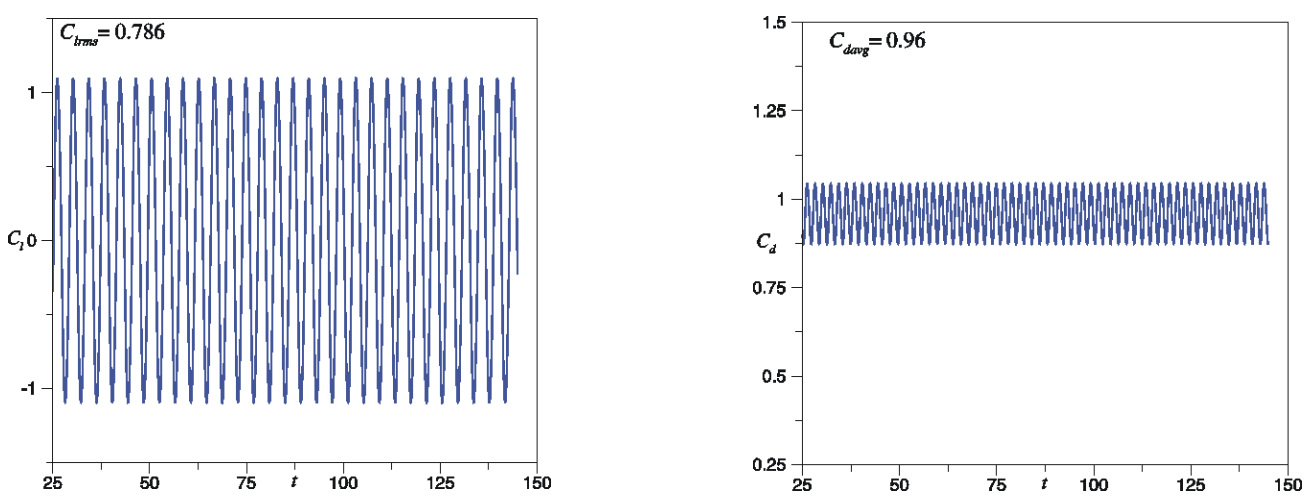

(c) SST Model
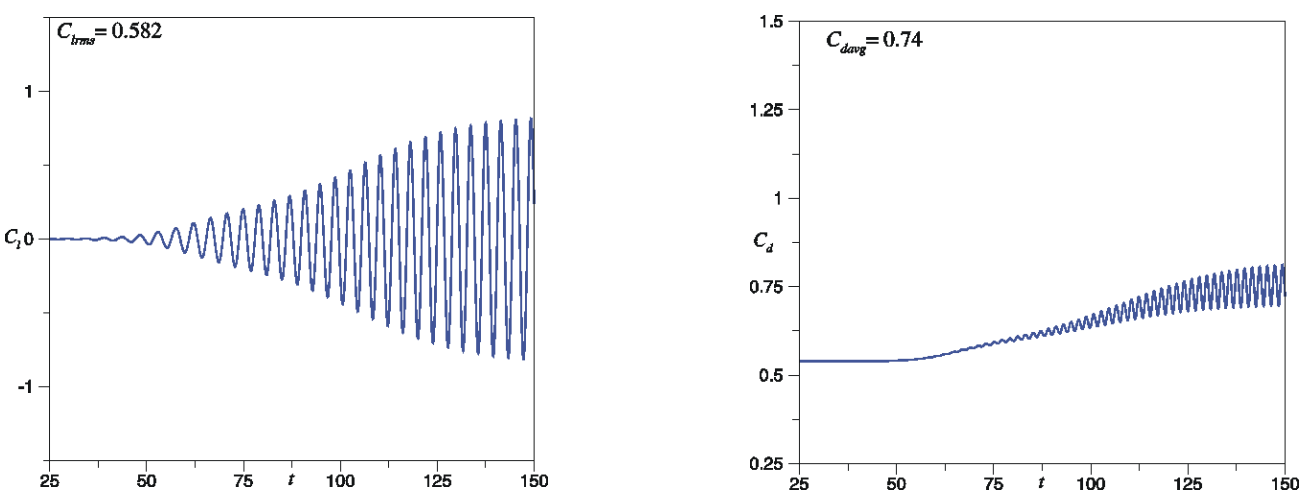

(d) SA Model

Fig. 3. Temporal variation of lift $\left(C_{l}\right)$ and drag $\left(C_{d}\right)$ coefficient using different turbulence models (Grid:121 $\times 144, \Delta t=0.05, R e=10^{5}$ ) 
B.N. Rajani et al. / JAFM, Vol. 5, No. 1, pp. 67-79, 2012.
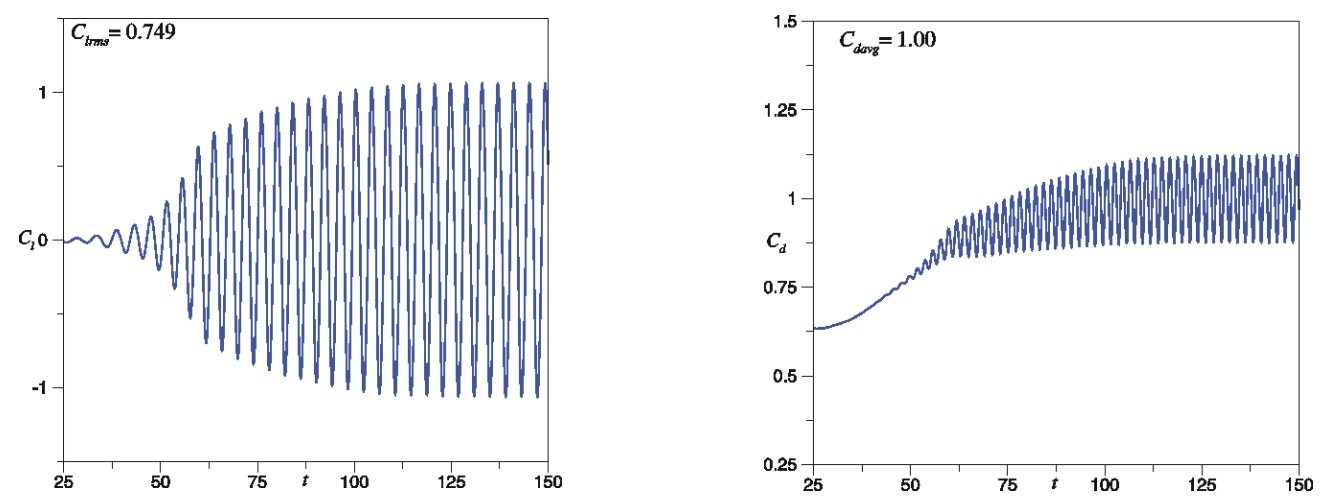

(a) $R e=10^{5}$
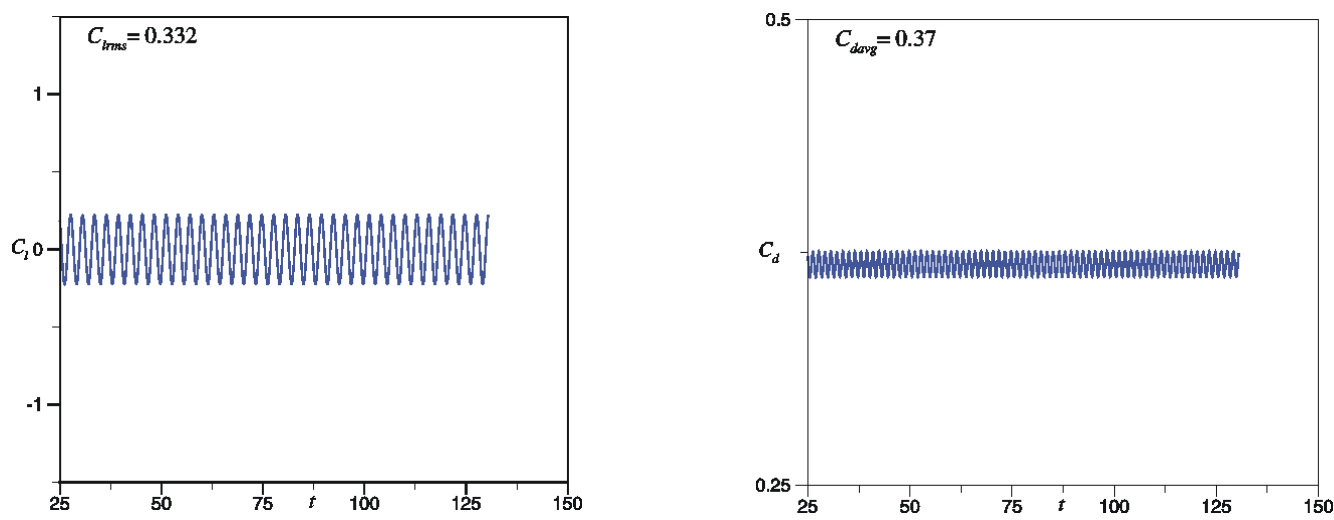

(b) $R e=8.5 \times 10^{5}$
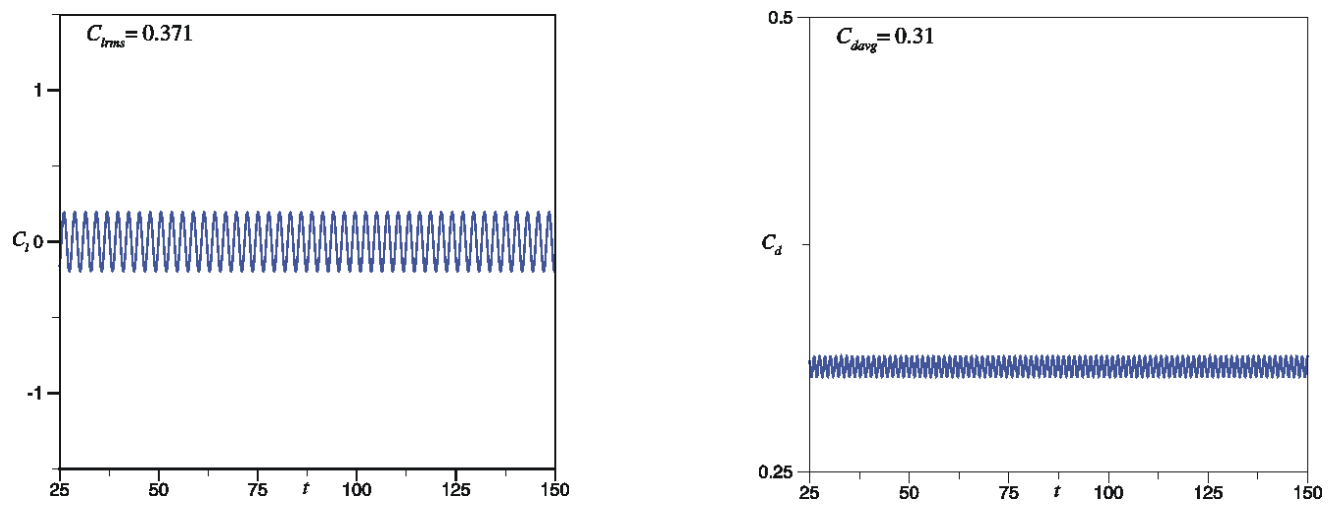

(c) $\operatorname{Re}=3.6 \times 10^{6}$
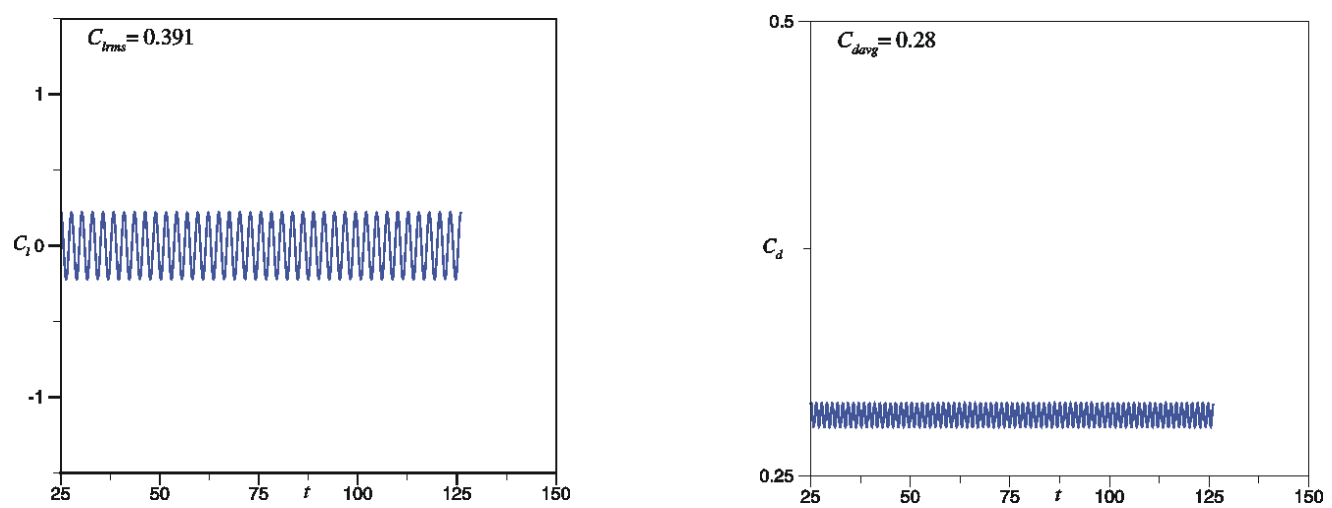

(d) $R e=10^{7}$

Fig. 4. Temporal variation of lift $\left(C_{l}\right)$ and $\operatorname{drag}\left(C_{d}\right)$ coefficient at different $R e$ using Chien low Re $k-\varepsilon$ turbulence model (Grid: $121 \times 144, \Delta t=0.05$ ) 

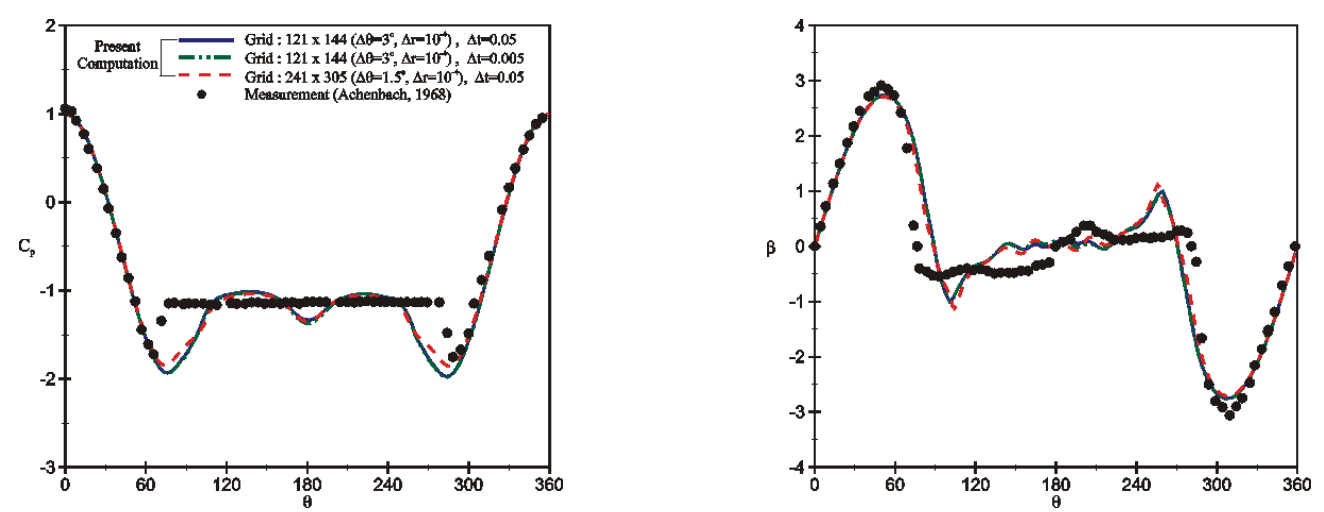

(a) $R e=10^{5}$
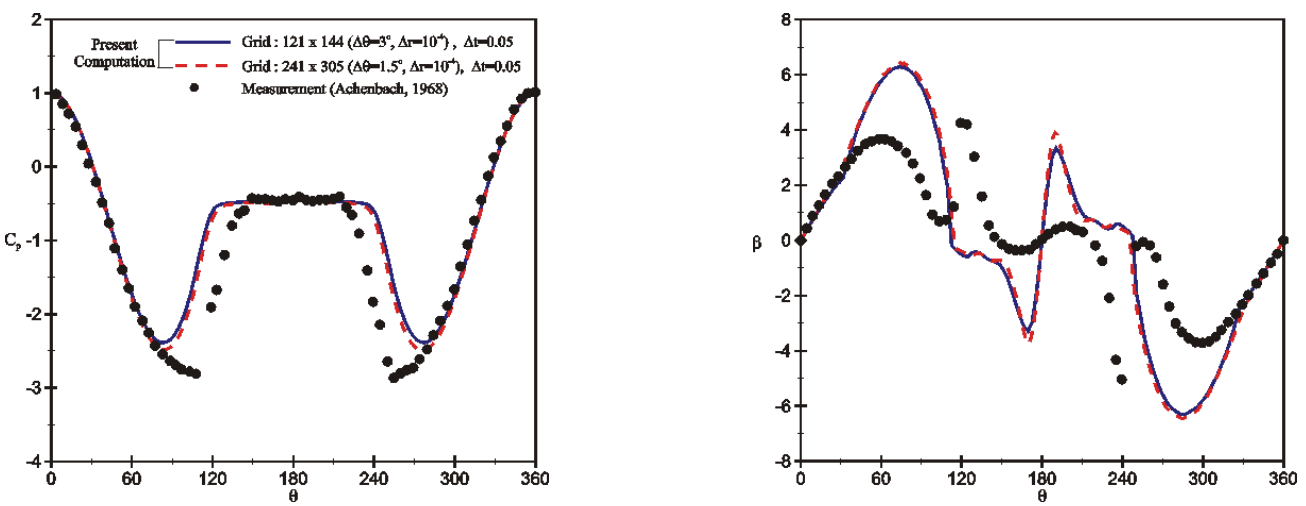

(b) $R e=8.5 \times 10^{5}$
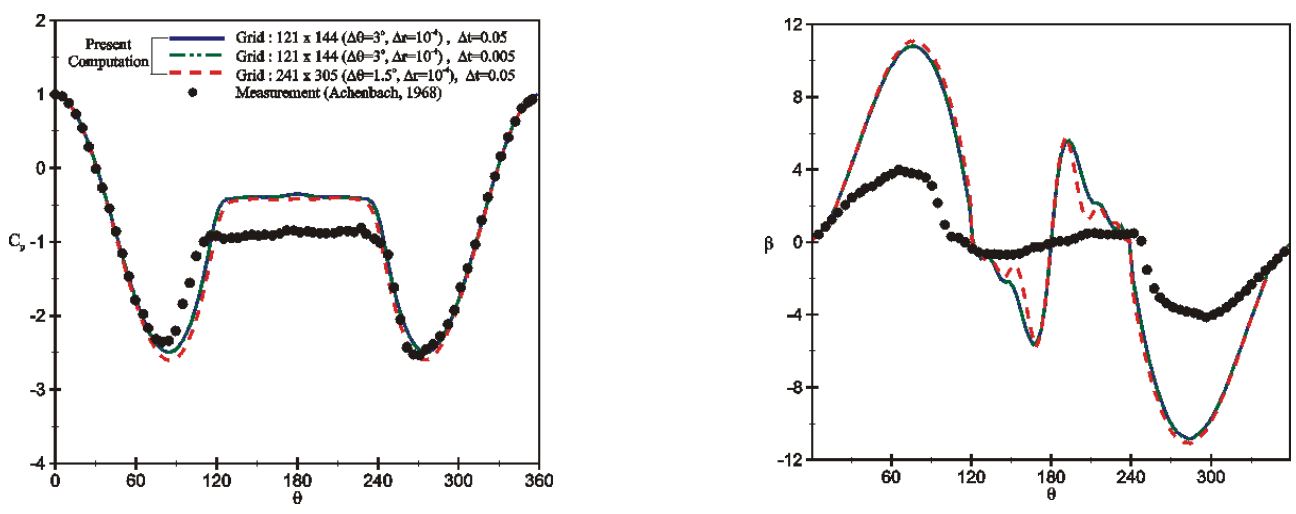

(c) $R e=3.6 \times 10^{6}$
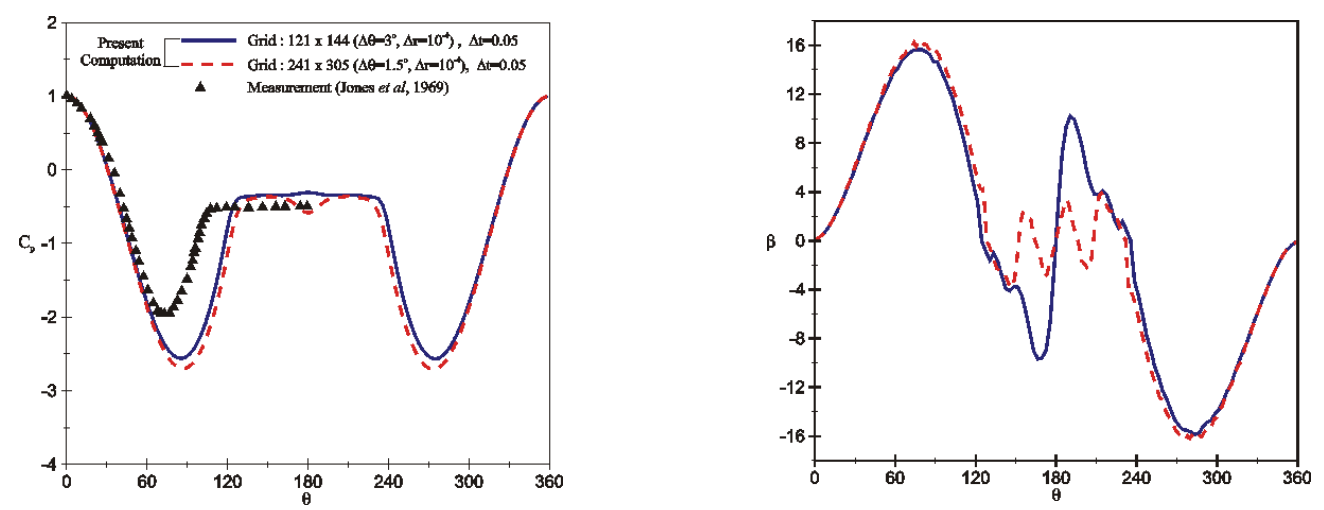

(d) $R e=10^{7}$

Fig. 5. Surface pressure $\left(C_{p}\right)$ and skin friction coefficient $(\beta)$ around the cylinder at different $R e$ using Chien's low $R e k^{-} \varepsilon$ turbulence model 

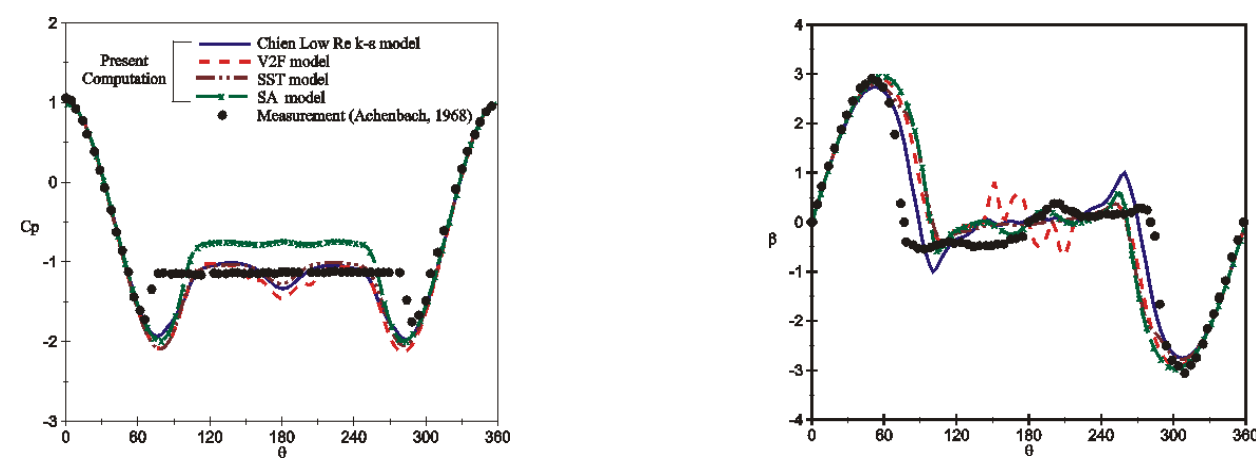

(a) $R e=10^{5}$
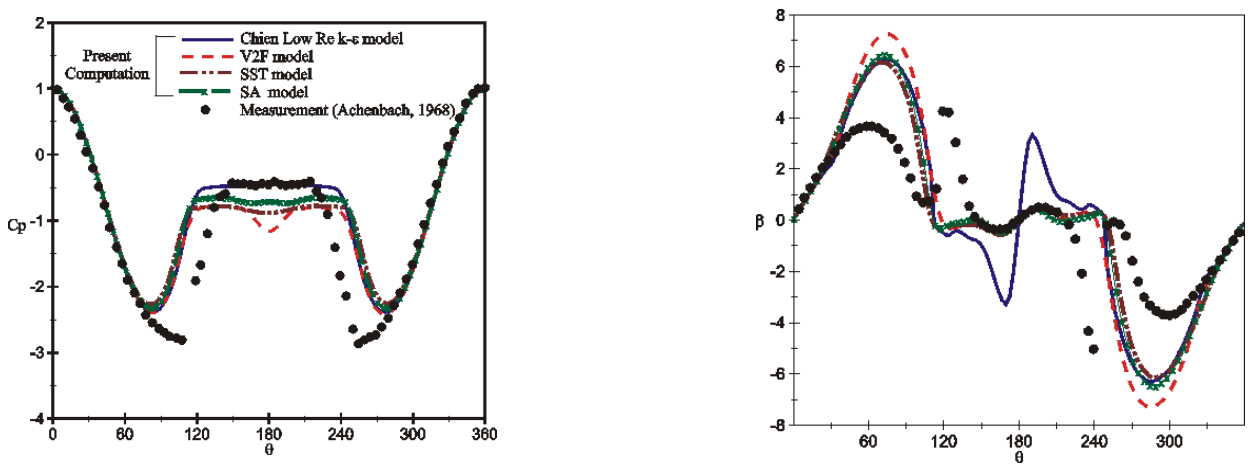

(b) $R e=8.5 \times 10^{5}$
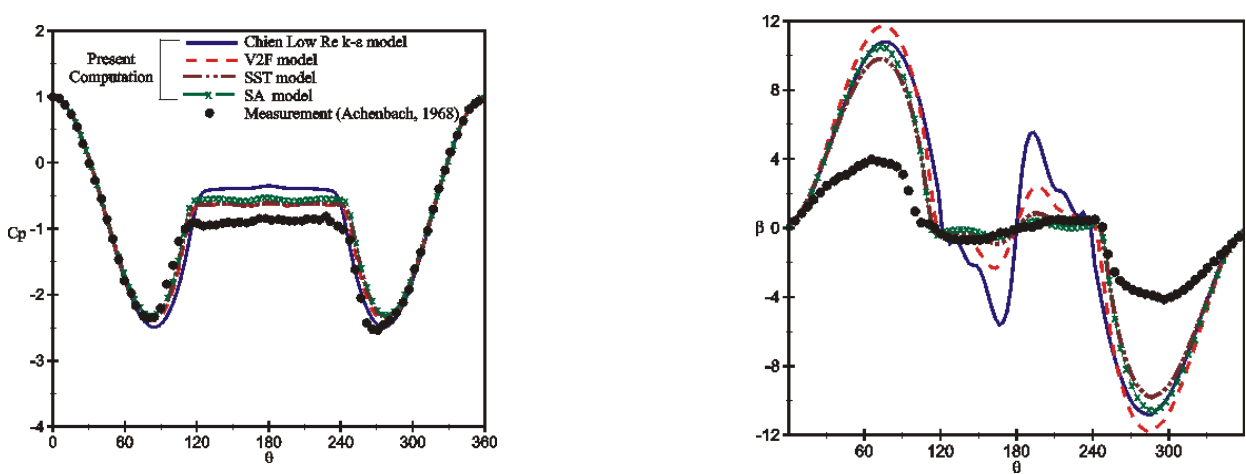

(c) $R e=3.6 \times 10^{6}$
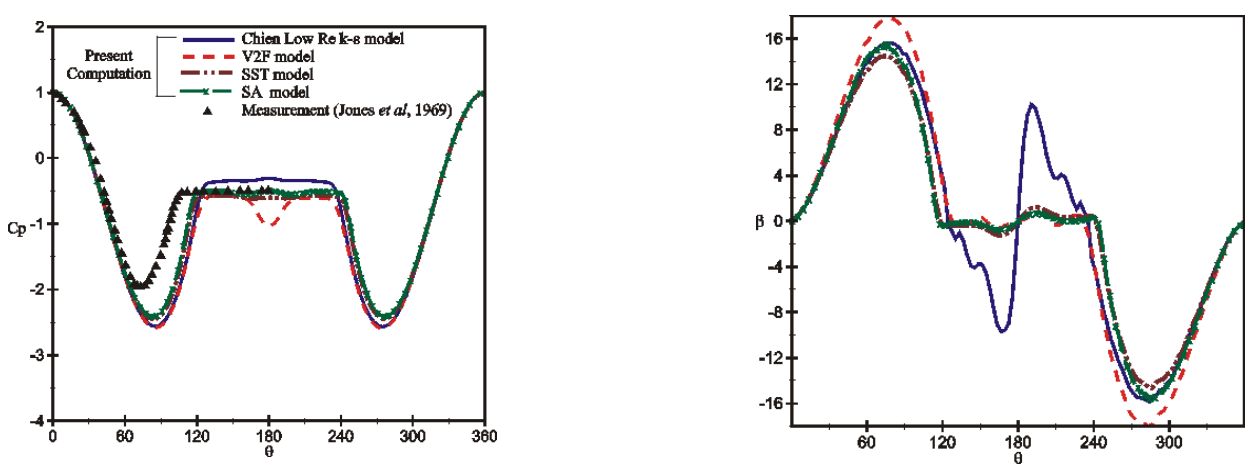

(d) $R e=10^{7}$

Fig. 6. Surface pressure $\left(C_{p}\right)$ and skin friction coefficient $(\beta)$ around the cylinder at different $R e$ using different turbulence models (Grid: $121 \times 144, \Delta t=0.05)$ 